\title{
Molecular and epidemiological characterization of Plasmodium vivax recurrent infections in southern Mexico
}

\author{
Lilia Gonzalez-Ceron ${ }^{1 *}$, Jianbing Mu², Frida Santillán ${ }^{1}$, Deirdre Joy², Marco A Sandoval', Gerardo Camas', \\ Xinzhuan $\mathrm{Su}^{2}$, Elena $\mathrm{V} \mathrm{Choy}^{1}$ and Rene Torreblanca ${ }^{3}$
}

\begin{abstract}
Background: In southern Mexico, malaria transmission is low, seasonal, and persistent. Because many patients are affected by two or more malaria episodes caused by Plasmodium vivax, we carried out a study to determine the timing, frequency, and genetic identity of recurrent malaria episodes in the region between 1998 and 2008.

Methods: Symptomatic patients with more than one P. vivax infection were followed up, and blood samples were collected from primary and recurrent infections. DNA extracted from infected blood samples was analyzed for restriction fragment length polymorphism (RFLP) in genes encoding csp and msp3a, as well as size variation in seven microsatellites.

Results: One hundred and forty six parasite samples were collected from 70 patients; of these, 65 patients had one recurrent infection, four had two, and one had three recurrent infections. The majority of recurrent infections occurred within one year of the primary infection, some of which were genetically homologous to the primary infection. As the genetic diversity in the background population was high, the probability of homologous re-infection was low and the homologous recurrences likely reflected relapses. These homologous recurrent infections generally had short ( $<6$ months) or long (6-12 months) intervals between the primary (PI) and recurrent (RI) infections; whereas infections containing heterologous genotypes had relatively longer intervals. The epidemiological data indicate that heterologous recurrences could be either relapse or re-infections.

Conclusions: Genetic and temporal analysis of $P$. vivax recurrence patterns in southern Mexico indicated that relapses play an important role in initiating malaria transmission each season. The manifestation of these infections during the active transmission season allowed the propagation of diverse hypnozoite genotypes. Both short- and long-interval relapses have contributed to parasite persistence and must be considered as targets of treatment for malaria elimination programs in the region to be successful.
\end{abstract}

Keywords: Plasmodium vivax, Hypnozoite, Relapse, Genetic homology, Epidemiology, Southern Mexico

\section{Background}

Plasmodium vivax is responsible for $99 \%$ of malaria cases in Mexico. In the 1980's more than 100,000 cases were reported each year. Due to intensive malaria control efforts carried out by the National Malaria Control Program in the 1990s, the numbers of cases have been declining ever since. Malaria transmission is general low and seasonal, and is concentrated in residual foci along

\footnotetext{
* Correspondence: Igonzal@insp.mx

'CRISP, National Institute for Public Health, Tapachula, Chiapas 30700, Mexico Full list of author information is available at the end of the article
}

the Pacific coast. In the past 10 years, $\sim 80 \%$ of malaria cases reported in Mexico have been from Oaxaca and Chiapas provinces $[1,2]$. These malaria foci are vulnerable to severe climate events as evidenced by malaria outbreaks that occurred coincident with "el Niño" and hurricane Paulina in 1998 in Oaxaca and Chiapas States, and Hurricane Stan at the end of 2005 in southern Chiapas.

P. vivax relapses are recurrent blood infections produced by latent parasites (hypnozoites) in the liver [3]. These episodes are of epidemiological significance, as

\section{() Biomed Central}

(c) 2013 Gonzalez-Ceron et al.; licensee BioMed Central Ltd. This is an Open Access article distributed under the terms of the Creative Commons Attribution License (http://creativecommons.org/licenses/by/2.0), which permits unrestricted use, distribution, and reproduction in any medium, provided the original work is properly cited. 
they contribute to the persistence of malaria transmission in affected regions, which may be particularly relevant where malaria transmission is seasonal. A person inoculated with $P$. vivax sporozoites has from 5 to $80 \%$ probability of producing latent hypnozoites after the primary attack [4]. These latent parasites are activated at variable latency periods by as yet unknown factors $[5,6]$. The relapse pattern comprises the latency period and number of relapse episodes; it may be influenced by parasite strain, transmission dynamics, sporozoite inoculation rate for different vector species, and host factors [5-8].

Primaquine $(\mathrm{PQ})$ is an 8 -aminoquinoline and is the only licensed antimalarial drug currently available that can be used to treat the dormant liver stage parasites (hypnozoites). PQ has been used to prevent $P$. vivax relapses in 1950 [9]. A 14-day PQ regimen $(0.25 \mathrm{mg}$ base/ $\mathrm{kg} /$ day) is currently recommended by the World Health Organization (WHO) [10]. In some $P$. vivax affected regions, parasites show reduced susceptibility to supervised PQ treatment, and higher PQ doses are required to reduce recurrence rates [11-13]. In Mexico, standard anti-malarial treatment guidelines included intermittent single doses of chloroquine (CQ) and PQ combination adjusted to age groups (from 10 and 0.75 for adults to 2.5 and $\sim 0.16 \mathrm{mg} / \mathrm{kg}$ for children, respectively) [14] as recommended by WHO [10], is administered once monthly for three months, then no treatment is given for the following three months. This treatment regimen is repeated up to six times for a total of three years to suppress recurrence onset $[14,15]$. Although this regimen has been administered for more than a decade, there is no baseline data describing the recurrence characteristics prevailing in southern Mexico. Currently, there is no evidence suggesting the presence of chloroquine resistant $P$. vivax in the region.

In recent years, Mexico has been in a pre-elimination phase due to significant reductions in malaria transmission [16]. It is now imperative to determine prevailing relapse patterns in the affected regions in order to support effective pre-elimination practices. Relapse patterns could evolve in response to changing drug pressure and transmission dynamics. The genetic identity of relapsing parasites is known to vary according to the transmission intensity [17-19]. In very low transmission settings most individuals are inoculated by no more than one infective mosquito bite per year, often carrying a single genotype infection, and the resulting primary and relapse episodes are mostly caused by the same parasite genotype (genetic homology) [17]. However, under more intense and complex transmission where mixed genotype infections and multiple infected bites are more common, many relapse episodes may in fact be produced by parasites with genotypes not initially detected in the primary infection (genetic heterology) [18,19]. Additionally, it has been proposed that relapse could be triggered by re-infection, leading to frequent infections of mixed genotypes [5]. In fact, one would predict a spectrum of homologous versus heterologous relapse rates across different transmission settings. In this report, molecular and epidemiological analyses of recurrent malaria infections were carried out in a malaria hypo-endemic region of southern Chiapas between 1998 and 2008 in order to understand the timing and molecular identity of recurrent $P$. vivax.

\section{Methods}

\section{Sample collection}

The study site (Tapachula and surrounding municipalities in southern Mexico) comprises two main sub-regions with alternating malaria transmission; in the temperate foothills transmission occurs mainly during January-June and in the coastal region during August-November. Symptomatic patients who consented to donate a capillary or venous blood sample were enrolled in the study. $P$. vivax infection was diagnosed by Giemsa-stained thick blood smears at the Regional Center for Research in Public Health (CRISP-INSP) during 1998-2008 [20-22]. Whole blood was stored in liquid nitrogen, and then transferred to Whatman No.2 filter paper (Whatman International, Ltd., Maidstone, England), dried and kept in the dark. After collection, filter paper samples were coded to block out identifying patient information, and part of the paper was used in DNA extraction and microsatellite analyses. All patients were treated by the local malaria control program according to the Mexican Malaria Treatment Guidelines [14]. Patients received anti-malarial treatment whether or not they agreed to give a blood sample. For the relapse study, both passive and active follow up were carried out. A second blood sample was collected from symptomatic patients who returned to CRISP-INSP seeking diagnosis and treatment of recurrent $P$. vivax infection (1998-2008). In addition, other patients were visited at their villages monthly to detect recurrent symptomatic infections (2005-2008). The majority of the analyses reported here were carried out on the paired blood samples with primary (PI) and recurrent (RI) infections from a single patient. However, we also included available non-paired samples from the region in the estimate of background microsatellite genetic diversity. The interval of infection-time between primary and recurrent infections is reported in weeks.

\section{Molecular analysis of $P$. vivax samples}

One hundred $\mu$ of infected blood or 6 punches of $5 \mathrm{~mm}$ diameter dried blood in filter paper were used to extract DNA using the QIAamp ${ }^{\circledR}$ DNA Blood Mini Kit (QIAGEN, Hilden, Germany) following the manufacturer's instructions. The DNA obtained was dissolved in water and stored at $-20^{\circ} \mathrm{C}$ until used. To determine the $P$. vivax 
genotypes, nine distinct markers (three marker sets) were analyzed, including variants in the circumsporozoite repeat region (cspr) [23] and merozoite surface protein 3 alpha domain $(m s p 3 \alpha)$ [24-26], and 7 microsatellites positioned on different chromosomes (Table 1) [27]. The cspr and $m s p 3 \alpha$ variants were genotyped by polymerase chain reaction and restriction fragment length polymorphism (PCRRFLP) [23]. The microsatellite markers were genotyped using capillary electrophoresis.

a) PCR-RFLP genotyping of the $c s p$ central repeat region (cspr). A nested PCR was carried out to amplify the complete cspr using primers CSP-1 (5'-cgcactgcgggca caatgtagatc-3') and CSP-2 (5'-ggttacactgcatggagtcc-3') for the first round amplification, and F-Pv9a (5' -gccaacgg tagctctaacttt-3') [28] and CSP-RI (5'-aataagctgaaacaacca$\left.3^{\prime}\right)$ for the second amplification. The PCR reaction mixed was prepared as follow: $2 \mu \mathrm{l} 10 \mathrm{X}$ Buffer, $2 \mathrm{mM}$ magnesium chloride, $0.2 \mathrm{mM}$ dNTPs (Invitrogen, 10297018 Carlsbad, CA.), $10 \mu \mathrm{M}$ each primer, $5 \mathrm{U}$ GoTaq ${ }^{\circledR}$ Flexi DNA polymerase (Promega, Madison WI) and approximately $100 \mathrm{ng}$ of genomic DNA in a $20 \mu \mathrm{l}$ final volume. Two $\mu$ l of the product from the first amplification was added to the second PCR of the same setup. The PCR conditions were as follow: first cycle at $95^{\circ} \mathrm{C}$ for $3 \mathrm{~min}$, followed by 35 cycles of $95^{\circ} \mathrm{C}$ for $30 \mathrm{sec}, 57^{\circ} \mathrm{C}$ (for first PCR) or $58^{\circ} \mathrm{C}$ (for second PCR) for $30 \mathrm{sec}$ and $72^{\circ} \mathrm{C}$ for $1 \mathrm{~min}$, and followed by a final extension at $72^{\circ} \mathrm{C}$ for 10 min using a MyCycler (Biorad, Hercules). The PCR products were digested with AluI (New England Biolabs, Beverly, MA) and BstI (Promega, Madison WI) which have restriction sites in the cspr encoding $\mathrm{Vk} 247$ and Vk210 regions, respectively [29]. DNA fragments were resolved in a $1.5 \%$ gel, visualized under a UV-transilluminator and photographed using the BioDoc-it ${ }^{\mathrm{TM}}$ digital photodocumentation system (UVP Inc, Upland, California).

b) PCR-RFLP genotyping of the $m s p 3 \alpha$ gene. The $m s p 3 \alpha$ gene was amplified in a nested PCR using the published primers (P1, P2, N1 and N2) and a modified version of a previously reported protocol [30]. The PCR was prepared as follow: $2 \mu \mathrm{l} 10 \mathrm{X}$ buffer, $1.875 \mathrm{mM}$ magnesium chloride, $0.5 \mathrm{mM}$ of dNTPs (Invitrogen, Carlsbad, CA.), 25 pM of each primer P1 and P2, $1.25 \mathrm{U}$ of GoTaq ${ }^{\circledR}$ Flexi DNA polymerase (Promega, Madison WI) and 1 or $5 \mu \mathrm{l}$ of genomic DNA extracted from whole or dried blood, respectively, in a final PCR volume of $20 \mu \mathrm{l}$. The first PCR was run at $95^{\circ} \mathrm{C}$ for $3 \mathrm{~min}$, followed by 35 cycles of $94^{\circ} \mathrm{C}$ for $60 \mathrm{sec}, 56^{\circ} \mathrm{C}$ for $60 \mathrm{sec}$ and $72{ }^{\circ} \mathrm{C}$ for $2.5 \mathrm{~min}$, and a final extension cycle at $72^{\circ} \mathrm{C}$ for $10 \mathrm{~min}$. The second round of PCR reaction was carried out with $\mathrm{N} 1$ and N2 primers using the same PCR reaction after addition of $2 \mu \mathrm{l}$ of first PCR product into a $20 \mu \mathrm{l}$ mixture. The reaction was run at $95^{\circ} \mathrm{C}$ for $2 \mathrm{~min}$, followed by 30 cycles of $94^{\circ} \mathrm{C}$ for $60 \mathrm{sec}, 62^{\circ} \mathrm{C}$ for $60 \mathrm{sec}$ and $72^{\circ} \mathrm{C}$ for $1.5 \mathrm{~min}$, and a final extension cycle at $72^{\circ} \mathrm{C}$ for $10 \mathrm{~min}$. For twenty-seven samples that failed to amplify, magnesium chloride and dNTPs were increased to $2.5 \mathrm{mM}$ and $1.0 \mathrm{mM}$, respectively, and PCR conditions were altered. A total of eight samples did not amplify under either PCR conditions. PCR products were digested with AluI (New England BioLabs, Beverly, MA), which was used to distinguish multiple $m s p 3 \alpha$ alleles for parasites from distinct geographic regions such as Papua New Guinea [30], India [31], Thailand [26], and Iran [23]. The digested paired DNA samples were separated in $2 \%$ agarose gels and stained with ethidium bromide and photographed.

c) Microsatellite (MS). Seven MS were also used to type recurrent infections to validate the capacity of the

Table $1 P$. vivax microsatellites and their primers used for PCR amplification and detection of nucleotide fragment length

\begin{tabular}{|c|c|c|c|c|}
\hline Microsatellite & Chromosome & Repeat unit & Primers & \\
\hline \multirow[t]{2}{*}{ MS 040} & 6 & AAAT & $\mathrm{F}$ & 6FAM-ATTTGCGTACGGTTAAGAT \\
\hline & & & $\mathrm{R}$ & CAGGGTTATTCAATTTGCT \\
\hline \multirow[t]{2}{*}{ MS 092} & 5 & GAA & $\mathrm{F}$ & 6FAM-TCACTGATCTTTTCGCATG \\
\hline & & & $\mathrm{R}$ & TAGTAGCATAGTGGTAGTA \\
\hline \multirow[t]{2}{*}{ MS 116} & 10 & GAA & $\mathrm{F}$ & NED-AAATGCAAGATCCAAGAAAT \\
\hline & & & $\mathrm{R}$ & GTCGCTCTTCATGTGGCA \\
\hline \multirow[t]{2}{*}{ MS 033} & 3 & CAT & $\mathrm{F}$ & 6FAM-CGATTCGTGCTATTTGC \\
\hline & & & $\mathrm{R}$ & CCTGCTACATATTTGGC \\
\hline \multirow[t]{2}{*}{ MS 176} & 14 & AT & $\mathrm{F}$ & 6FAM-ATAATGGCGTCATCCTTCA \\
\hline & & & $\mathrm{R}$ & TTCAGCATGCGCTGTTTAT \\
\hline \multirow[t]{2}{*}{ MS 315} & 3 & AT & $\mathrm{F}$ & NED-TTAACGGTTAATCCTCTATT \\
\hline & & & $\mathrm{R}$ & TTGTGTCTATTTGGCCATT \\
\hline \multirow[t]{2}{*}{ MS 206} & 12 & $\mathrm{GTT}$ & $\mathrm{F}$ & 6FAM-TCTITATGTTGTACTGCTC \\
\hline & & & $\mathrm{R}$ & ACCACTTACAAAAGTGTGA \\
\hline
\end{tabular}


combined genotype cspr-msp3 $\alpha$ to discriminate heterologous infections, because MS are generally more variable than nucleotide substitutions. In addition, 236 non-recurrent samples collected during the same time period were also typed using the same MS in order to provide background diversity and marker performance estimates. Samples were scored as mixed genotypes if a MS marker produced a minor peak $\geq 33 \%$ of the primary peak. MS primers are listed in Table 1. Due to limited DNA quantity or quality for some samples, we were not able to obtain microsatellite information for all the samples.

\section{Data analysis}

Malaria cases reported by the Sanitary Jurisdiction VII during 1998-2007 were compiled in order to calculate the annual parasitic index, defined as number of cases/ 1,000 individuals (from affected villages)/per year. From this we estimated the number of cases/1,000 individuals/ 2-month intervals in order to compare rates between high and low transmission periods. The case report includes all cases confirmed by microscopic analysis of thick smears from all institutions of the corresponding jurisdiction. To characterize the recurrent patterns in the study region, a database was compiled to identify the number of malaria episodes per person indicating name, gender, age, village, municipality of origin and previous malaria infections (self-reported or by a family member). The number of malaria cases diagnosed per two-month period was plotted to simulate transmission dynamics and to identify intervals with minimum risk of reinfection that could be used to identify putative heterologous relapse episodes. For patients identified as having more than one infection, the time-interval between primary and recurrent infections was estimated starting with the day the sample was collected for $P$. vivax diagnosis. Once malaria episodes were associated with individual patients, all samples were coded and all the patient information was masked. The original database was secured at the study investigator's office. Patients registered between 2005 and 2008 as having more than one episode were confirmed by a home-visit.

For putative relapse identification, blood samples from patients with more than one episode and with good genotype data, comprising at least cspr and $m s p 3 \alpha$ genotypes, and/or 6-7 MS, and the malaria epidemiology data were used. Recurrent infections showing similar cspr and $m s p 3 \alpha$ genotypes as the primary infections were defined as homologous. The correspondence between cspr-msp3 $\alpha$ and MS heterology vs homology was determined. Heterologous recurrent infections detected when transmission was low or non-existent (based on malaria case records) may in fact be heterologous relapse. Pairs of samples with mixed genotype infections detected in either the primary or recurrent infection were not included in the analysis. Relapse episodes were grouped according to their time as follows: 1) early (126 weeks), long (27-52 weeks) [32], or late (> 52 weeks). We calculated the ratio of the number of homologous versus heterologous recurrences within 52 weeks and > 52 weeks. All microsatellite analyses were carried out as described [27] using GenAlEx v 6.5 [33].

All MS analyses were blinded to the relationship between samples and their origins. The baseline population diversity was derived from MS genotype data from 326 non-recurrent infections collected during the 10 year study period. The seven MS loci were used in combination to calculate the likelihood that two infections have the same seven-locus genotype by chance by taking the product of the individual homozygosities [34]. Chi-square and t-test (at 95\% confidence) were carried out in STATA 9.0 (Stata Corporation LD).

\section{Ethical approval}

The protocol for sample collection was approved by the Ethics Committee of the National Institute of Public Health (INSP), Mexico. Written informed consent was obtained from all patient and/or their guardians for patients below 18 years old.

\section{Results}

Transmission dynamics and temporal pattern of recurrent malaria cases compiled from the study region during 1998-2007

During this period, the annual parasitic index (API) fluctuated between 0.58 and 3.4, and between 0.25 and 2 for 1,000 individuals living in the affected villages or the entire municipality, respectively. The minimal number of malaria cases reported per 1,000 individuals/2 months ranged from 0.031 to 0.41 across all years. In contrast, the highest transmission levels occurred during the dry season, with between 0.43 and 1.9 cases per 1,000 individuals/ 2 months. We therefore set a high transmission lower bound cut-off at $\geq 0.4$ cases $/ 1,000$ individuals $/ 2$ month across the 10 yearperiod. From 7,694 malaria reports included in the database, $8.1 \%(n=626)$ of patients were identified as having one or more recurrent $P$. vivax infections. None of the recurrences occurred within 28 days (CQ surveillance period); all recurrences occurred from 5 weeks to several years after the primary infection. Among those, $25.2 \%$ occurred $\leq 26$ weeks, 46.3\% occurred within 27 and 52 weeks, and $28.4 \%$ occurred $\geq 105$ weeks after the primary infection (Figure 1). From the total recurrent infections, $88.2 \%$ reported two malaria episodes, $9.7 \%$ had three malaria episodes, and only a few patients had three to six recurrent episodes.

\section{cspr-msp3a genotypes and genetic diversity}

Genotypes from 146 blood samples collected from 70 patients with more than one infection were scored based 
on the three marker sets to determine whether recurrent infections contained the same genotype(s) as the primary infection (PI), as well as to detect mixed genotype infections (Additional file 1). Among these patients, 65 had one recurrent infection (RI), four had two RI, and one had 3 RI. The cspr genotype was determined for all but two samples (one pair with insufficient sample) (Table 2). RFLP-msp $3 \alpha$ genotypes were successfully obtained for 117 paired samples from a subset of 56 patients. A conserved $\sim 2 \mathrm{~kb} m s p 3 \alpha$ product was amplified in all these samples except from patient 58 that had a $\sim 1.5 \mathrm{~kb}$ fragment. Two cspr genotypes (corresponding to vk210 and vk247) and eight $m s p 3 \alpha$ genotypes were identified (Figure 2), and a total of 113 single $P$. vivax $m s p 3 \alpha$ genotype infections were detected. Among the eight $m s p 3 \alpha$ genotypes, genotype $\mathrm{A}, \mathrm{B}$, and $\mathrm{C}$ comprised the majority (85.8\%). All samples with the $m s p 3 \alpha-\mathrm{A}$ genotype also had the cspr vk247 genotype. Samples with $m s p 3 \alpha-\mathrm{B}$ or $m s p 3 \alpha-\mathrm{C}$ had either $c s p r$ vk210 or vk247 genotypes. There were five other less common $m s p 3 \alpha$ genotypes; all have cspr vk210. Genotype D was detected in both PI and RI of two patients; E was detected in PI and RI of three patients; whereas G and J were detected in PI and RI from a single patient each (patient 58 and 59 respectively). Also, both samples from patient 58 had a unique $m s p 3 \alpha$ genotype $(\mathrm{H})$ with a fragment size of 1.5 $\mathrm{kb}$. These rare genotypes did not cluster in time but rather were collected throughout the 10-year sampling period as were the more common genotypes. Given the

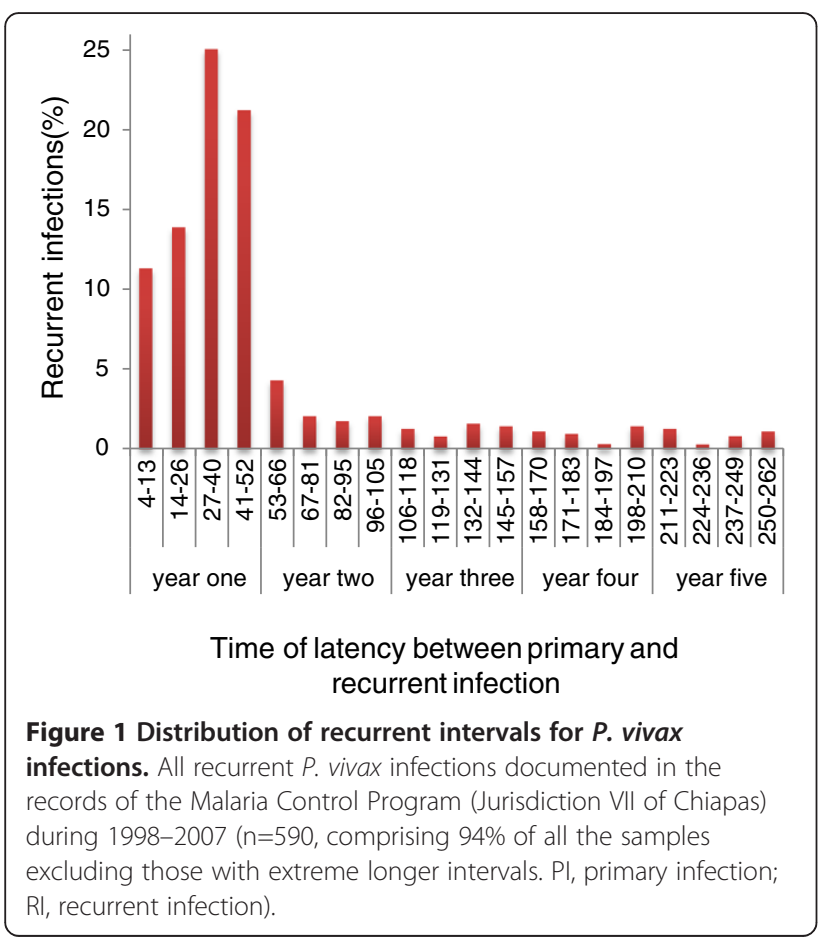

Table 2 Sample size, performance measures and diversity parameters for the nine molecular markers used to type the recurrent sample pairs used in this study

\begin{tabular}{llllll}
\hline Marker & $\boldsymbol{N}$ & GFR (\%) & $\boldsymbol{N}_{\mathrm{a}}$ & \% polyclonal & $\boldsymbol{H}_{\mathrm{E}}$ \\
\hline & $(\mathrm{PI} / \mathrm{RI})$ & $(\mathrm{PI} / \mathrm{RI})$ & $(\mathrm{PI} / \mathrm{RI})$ & $(\mathrm{PI} / \mathrm{RI})$ & $(\mathrm{PI} / \mathrm{RI})$ \\
msp3a & $49 / 56$ & $27.1 / 25.3$ & $8 / 8$ & $5.7 / 5.3$ & $0.743 / 0.739$ \\
csp repeat & $69 / 74$ & $1.4 / 1.3$ & $2 / 2$ & $5.7 / 5.3$ & $0.492 / 0.505$ \\
MS 40 & $62 / 69$ & $0 / 0$ & $5 / 5$ & $0 / 0$ & $0.616 / 0.59$ \\
MS 92 & $62 / 69$ & $0 / 0$ & $8 / 9$ & $1.9 / 1.9$ & $0.754 / 0.806$ \\
MS 116 & $61 / 68$ & $1.6 / 1.4$ & $12 / 11$ & $0 / 0$ & $0.822 / 0.744$ \\
MS 33 & $58 / 69$ & $6.5 / 0$ & $7 / 8$ & $3.7 / 1.9$ & $0.706 / 0.63$ \\
MS 176 & $49 / 63$ & $21.0 / 8.8$ & $6 / 6$ & $0 / 0$ & $0.692 / 0.747$ \\
MS 315 & $59 / 67$ & $4.8 / 2.9$ & $5 / 5$ & $0 / 3.8$ & $0.593 / 0.62$ \\
MS 206 & $50 / 57$ & $19.4 / 17.4$ & $10 / 10$ & $0 / 0$ & $0.825 / 0.831$ \\
\hline
\end{tabular}

$P l$, primary infection; $R l$, recurrent infection.

$N$, number of samples; GFR, genotype failure rate, $N_{a}$, number of alleles, $\mathrm{H}_{\mathrm{E}}$, effective heterozygosity.

rarity of these less common genotypes, it is likely that their presence in both the PI and RI infections of individual patients was due to relapse episodes.

\section{Microsatellite variation}

Among the recurrent sample pairs, a subset of 62 primary (PI) and 69 recurrent (RI) infections were successfully genotyped using MS markers (Table 2 and Additional file 1). In addition, 326 non-recurrent samples were genotyped at 4-7 MS markers to obtain baseline information on population diversity (Table 3). Of these, 312 were successfully typed for all microsatellite markers. The number of alleles ranged from 6-15 for the baseline samples and from 5-12 for the recurrent samples (Tables 3 and 2). Effective heterozygosity $\left(H_{\mathrm{e}}\right)$ for the seven MS used in this study ranged from 0.555 0.818 (Table 3). MS $H_{\mathrm{e}}$ declined slightly over the 10 year period (Figure 3A). Given the decline in the number of malaria cases in the region starting in 2001, it is perhaps surprising that diversity did not show a steeper decline in later years, although this may be explained by an increase in sample size (Figure 3). When the seven MS loci are used in combination as we have done here, the likelihood that two infections have the same seven-locus genotype by chance is negligible $\left(\pi \mathrm{P}_{i}=1.39 \times 10^{-4}\right)$. Indeed, more than one MS haplotype could be found in parasite isolates with identical csp-msp3 $\alpha$ genotypes. For most of the patients with rare $m s p 3 \alpha$ genotypes $\mathrm{D}, \mathrm{E}$ and $\mathrm{G}$ (patient 7, 23, 38, 43, 46 and 66), where relapse rather than re-infection was suspected, heterologous microsatellite alleles were found in the RI samples. Two of six patient samples had one new microsatellite allele in the RI (patients 23 and 43), they also had low 

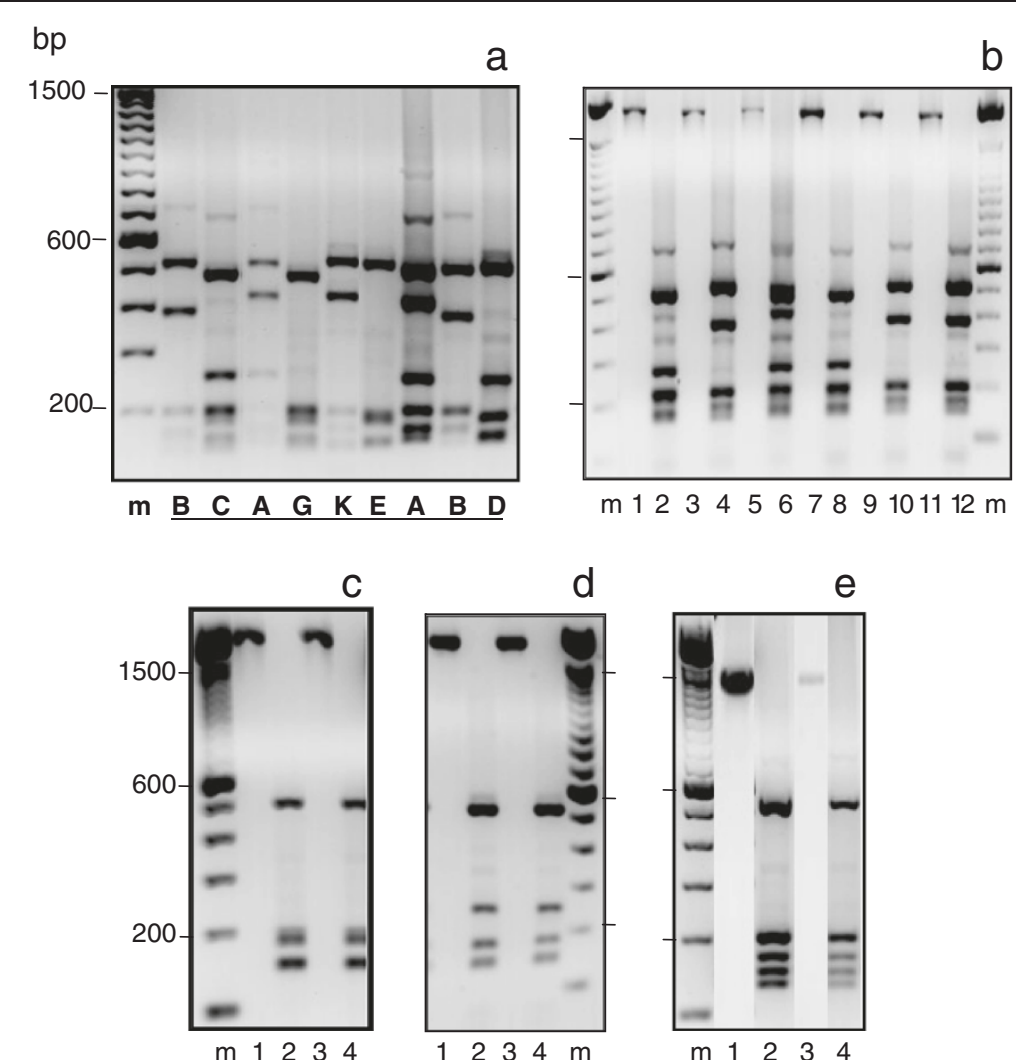

Figure 2 Plasmodium vivax msp3a genotypes. PCR products were digested with Alu I, and the digested products are separated side-by-side with their undigested products on agarose gels. a) Shows different genotypes indicated by capital letters underneath. b) Lanes 1, 3, 5, 7, 9, and 11 are undigested products of $\sim 2.0 \mathrm{~kb}$, and lanes $2,4,6,8,10$, and 12 were the same products digested with Alu I. Lane 2 presents genotype C; lane 4, 10, and 12 present genotype B; lane 6 and 8 are mixed genotypes of $A+C ; \mathbf{c}$ ) genotype $G$; d) genotype $D$; and e) genotype H. m, 100 bp DNA ladder.

frequent MS alleles (Additional file 1). If we define these pairs of infections as homologous, based on the rare but identical $m s p 3 \alpha$ alleles and that $1-2$ single unit differences in microsatellite alleles between infections could be due to DNA replication slippage within the patient.

Table 3 Sample size, performance measures and diversity parameters for the seven microsatellite markers used to type 326 baseline, non-recurrent samples collected between 1998 and 2007

\begin{tabular}{llllll}
\hline Marker & $\boldsymbol{N}$ & GFR (\%) & $\boldsymbol{N}_{\mathrm{a}}$ & \% polyclonal & $\boldsymbol{H}_{\mathrm{E}}$ \\
\hline MS 40 & 321 & 1.5 & 6 & 2.15 & 0.555 \\
MS 92 & 325 & 0.3 & 11 & 1.2 & 0.743 \\
MS 116 & 326 & 0 & 15 & 0.8 & 0.762 \\
MS 33 & 326 & 0 & 12 & 1.2 & 0.686 \\
MS 176 & 319 & 2.2 & 8 & 0.0 & 0.665 \\
MS 315 & 324 & 0.6 & 8 & 1.2 & 0.63 \\
MS 206 & 323 & 0.9 & 15 & - & 0.818
\end{tabular}

$N$, number of samples; GFR, genotype failure rate, $N_{a}$, number of alleles, $\mathrm{H}_{\mathrm{E}}$, effective heterozygosity.

\section{Relapse criteria based on microsatellite and csp-msp3} genotypes

Thirty nine paired samples produced genetic marker information for all three genetic marker sets. Among the 39 pairs, ten had heterologous cspr and/or $m s p 3 \alpha$ genotypes and all seven of them had three or more heterologous MS (patients 28, 29, 30, 37, 63, 65, 67), another one had two of six heterologous MS (patient 53) and only two pairs had 5 of 6 homologous microsatellites (patients 3 and 18) (Figure 4, Table 4 and Additional file 1). In contrast, 29 patients had the same cspr-msp $3 \alpha$ genotype and $>80 \%$ homology of six or seven MS. In particular, 14 pairs of samples (patients $6,7,9,17,20$, $21,34,36,38,45,46,61,63 \mathrm{RI} 2,66$ ) had the same alleles across all three-marker sets. However, four sample pairs with homogeneous cspr-msp $3 \alpha$ genotypes had two of six (patient 25), or two of seven (patients 8, 19RI1, 42) heterologous MS. A single patient with homogeneous cspr-msp $3 \alpha$ genotypes had three of six heterologous MS (patient 59). In spite of this, high agreement across marker sets suggests a low margin of error in accepting 

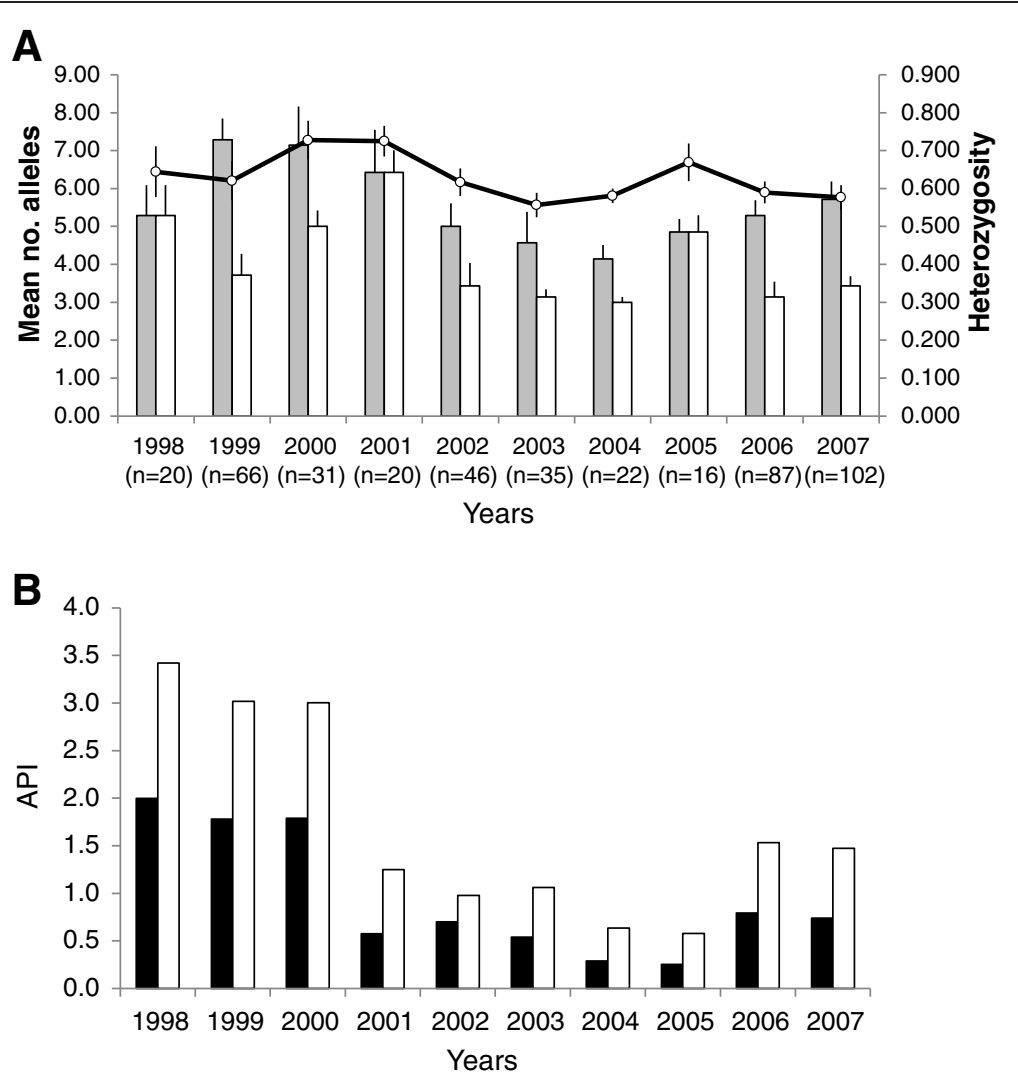

Figure 3 Microsatellite diversity and Plasmodium vivax infection rate in southern Mexico during a 10-year period. A. Pattern of allelic variation for 7 microsatellite loci using 474 bloodspot samples collected over 10 years. Grey, mean number of alleles (Na); white, mean number of alleles $\geq 5 \%$; black line, mean effective heterozygosity $\left(H_{e}=[n /(n-1)] \times 1-\sum p_{i}^{2}\right)$, shows a slight but persistent decrease over the time frame $\left(R^{2}=\right.$ 0.3109). B. Number of malaria cases per 1,000 people during a 10 year period across the entire study area (black bars), and within villages with positive malaria cases only (white bars). API: number of cases per year per 1,000 people.

homology by either the same cspr-msp3genotypes and/ or microsatellites (Chi-square; $p=0.003$ ).

\section{More homologous genotypes from patients with short interval between $\mathrm{PI}$ and $\mathrm{RI}$}

The average duration between PI and RI for those homologous RIs (by cspr/msp $3 \alpha / M S$; $\mathrm{n}=22$ ) was $41.5 \pm 44.3$ weeks, which was significantly different from those classified as heterologous RIs by cspr and/or $m s p 3 \alpha$ (128.4 \pm 104 weeks; $\mathrm{n}=10)(t$-test; $p=0.0022)$. Indeed, sample pairs with the same cspr-msp $3 \alpha$ genotypes and a high degree of homology among microsatellite markers also had shorter recurrent intervals than those with heterologous $m s p 3 \alpha$ or microsatellites (Figure 4, Table 4 and Additional file 1$)$. The majority $(76.6 \%)$ of paired samples with a latency period between five and 52 weeks were considered genetically homologous. The frequency of homologous recurrences declined gradually as the time interval between infections increased to 53-104 weeks and greater (total of 17.4\%), (Table 4). The ratio of the number of homologous versus heterologous recurrences was higher within 52 weeks (4.5) than > 52 weeks $(0.35)$
(Table 5). Homologous recurrent episodes occurred in people of both sexes and all ages, and originated from different locations throughout the transmission area (Additional files 1 and 2).

We observed more than two episodes of $P$. vivax malaria during the study period in five patients, including two patients who suffered three symptomatic malaria episodes (patients 19 and 43; Additional file 1). In both cases, parasites collected from all three episodes appear to be homologous based on our criteria for the marker set, although for patient 43 some data was missing (this episode was not included in the previous analysis) (Additional file 1). These putative homologous infections had latency periods of 27 and 43 weeks (patient 43 ), and 43 and 60 weeks (patient19). Interestingly, patient 51, also with three infections, showed a mixed PI and two different RI at 34 and 46 week intervals. Although MS data was not obtained for the PI, based on our criteria for cspr-msp $3 \alpha$, the first but not the second R1 was likely homologous to the PI. Patient 5 suffered 4 malaria episodes, two in 2002 with the same cspr-msp3 $\alpha$ genotype (13 weeks apart), and the other two episodes 


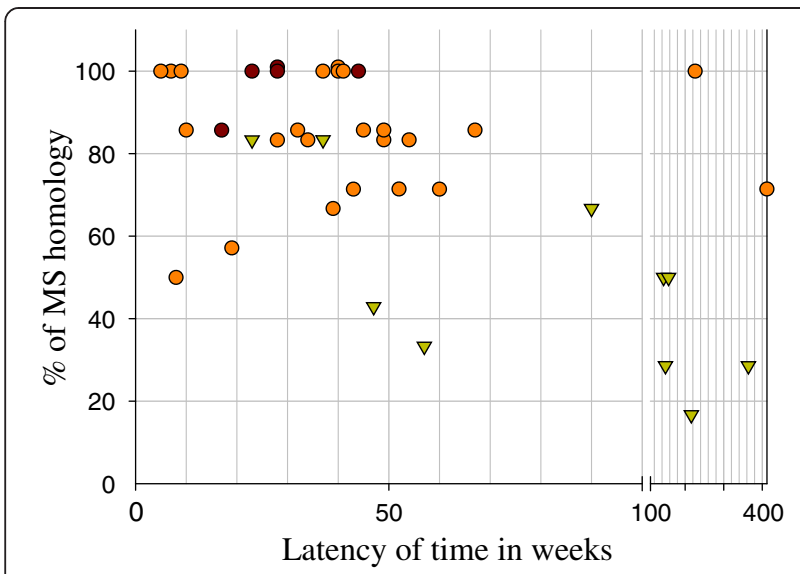

Figure 4 Concordance between $P$. vivax cspr-msp3a and microsatellite patterns for primary and recurrent infections with different latency periods ( $\mathrm{n}=39$ pair samples). Each data point indicates one sample pair (PI and RI). Circles indicate paired infections with homologous cspr-msp3a genotypes. Brown circles, rare msp3a genotypes $(\mathrm{D}, \mathrm{E}, \mathrm{G})$; orange circles, common msp3a genotypes (A, B, C). Green triangles indicate paired infections with heterologous cspr-msp3a genotypes.

in 2004 and 2005 (15 weeks apart); the third and fourth episodes were identical to each other but differed from the first and second episodes in their $m s p 3 \alpha$ alleles (B versus $\mathrm{A})$. There were also six polyclonal infections detected by cspr-msp $3 \alpha$ in the PI only (patients 10, 26, $32,35,51,70)$ that corresponded to $75 \%$ of the total detected, while the other two (25\%) were in the RI only (patients 2, 52) (Additional file 1).

According to the transmission intensity estimated for the study region, $71.4 \%$ (50 of 70 patients) of primary infections occurred during the high transmission season. In areas of seasonal transmission, relapses can occur immediately prior to the initiation of malaria transmission each year or during active transmission [35-38]. We observed both incidences in our study. There were no differences in the ratios between the number of homologous and heterologous recurrences during low versus high transmission seasons $(p=0.957)$ (Table 5) and were similarly distributed in the region (Additional file 3). In addition, no obvious correlation was observed between any genotypes and latency period; the $m s p 3 \alpha \mathrm{A}, \mathrm{B}$ and $\mathrm{C}$ genotypes were found in relapses of short (5-26 weeks), long (27-52 weeks) or even late (over 52 weeks) latency periods. Similarly, patients infected with low frequency genotypes cspr vk210 and $m s p 3 \alpha-\mathrm{D}, \mathrm{E}, \mathrm{G}, \mathrm{H}$ and $\mathrm{J}$ showed recurrence within 17-44 weeks, although the relatively shorter latency periods were likely associated with homologous relapses, compared with heterologous recurrences (relapse or re-infections). Heterologous and mixed genotype recurrent infections occurring during the lowest transmission season are likely relapses (e.g. patients 1, 2, 3 and 5).

\section{Discussion}

Relapse episodes can be caused by parasites that are either homologous or heterologous to the primary infections. If a patient is infected with a single parasite genotype, the relapse will have the same genotype as the PI [17]. If, however, a patient is infected with more than one parasite genotype, the relapse could result from any one of those genotypes. Many factors may contribute to the latency and frequency of relapse, including the number of sporozoites inoculated, host immunity, co-infection with other parasites such as Plasmodium falciparum, drug treatment, and the genetic composition of $P$. vivax strains [5].

A better understanding of the mechanism of relapse and the development of methods to distinguish relapse from new infections or recrudescence will greatly facilitate efforts to control and eliminate $P$. vivax malaria. PQ is currently the only licensed drug that has been shown

Table 4 Molecular classification of recurrent $P$. vivax carrying single genotype infections

\begin{tabular}{|c|c|c|c|c|c|c|}
\hline \multirow{2}{*}{$\begin{array}{l}\text { Interval time } \\
\text { in weeks }\end{array}$} & \multicolumn{4}{|c|}{ Genetic homology: } & \multirow{2}{*}{$\begin{array}{l}\text { Genetic heterology: } \\
\text { By any of the three } \\
\text { set of markers }(\mathrm{N}) \\
\text { Subtotal }\end{array}$} & \multirow[t]{2}{*}{$\mathrm{N}$ total } \\
\hline & $\begin{array}{l}\text { By all three } \\
\text { markers }(\mathrm{N})\end{array}$ & $\begin{array}{l}\text { *By cspr } \\
+m s p 3 a \\
\text { (N) }\end{array}$ & $\begin{array}{l}{ }^{*} \text { By MS } \\
\text { (N) }\end{array}$ & Subtotal & & \\
\hline $5-13$ & 4 & 2 & 1 & 7 & $1^{* *}$ & 8 \\
\hline $14-26$ & 2 & 1 & - & 3 & 3 & 6 \\
\hline $27-40$ & 9 & - & 7 & 16 & 3 & 19 \\
\hline $41-52$ & 5 & 1 & 3 & 9 & 3 & 12 \\
\hline $53-69$ & 2 & - & - & 2 & 3 & 5 \\
\hline 70-104 & 1 & - & - & 1 & 2 & 3 \\
\hline$\geq 105$ & 1 & - & - & 1 & 12 & 13 \\
\hline $\mathrm{N}$ total & 24 & 4 & 11 & 39 & 27 & 66 \\
\hline
\end{tabular}

*only these marker results were obtained; ${ }^{* *} m s p 3 a$ genotype $\mathrm{J} ; \mathrm{N}$, number of recurrent infections. All markers correspond to csp (circumsporozoite gene), msp3a (merozoite surface protein $3 a$ gene) and MS (microsatellite). Time-interval of homologous paired samples of rare msp3a genotypes (D, E, G, H, J) were within 14 and 52 weeks. 


\begin{tabular}{|c|c|c|c|c|}
\hline Group & \# Hom & \#Het & Ratio & *P-value \\
\hline PI_LTS & 16 & 8 & 2.00 & 0.957 \\
\hline PI_HTS & 35 & 18 & 1.94 & \\
\hline $\mathrm{Rl}<52 \mathrm{~W}$ & 41 & 9 & 4.50 & 0.0001 \\
\hline $\mathrm{Rl}>52 \mathrm{~W}$ & 6 & 17 & 0.35 & \\
\hline
\end{tabular}

Hom, homologous infections; Het, heterologous infections;

Ratio, ratio of homologous infection over heterologous infection;

PI_LTS, Primary infection during the low transmission season; PI_HTS, primary.

infection during the high transmission season; $\mathrm{RI}<52 \mathrm{~W}$, recurrent infection

within 52 weeks of the primary infection; RI $>52 \mathrm{~W}$, recurrent infection at or

after 52 weeks.

following the primary infection.

* Chi-square; IC 95\%.

to be effective in treating hypnozoites to prevent relapse, making treating $P$. vivax malaria and interrupting transmission more difficult. In southern Mexico, the current antimalarial guideline is designed to suppress recurrent infections only if the drugs are administered to patients at the onset of the blood infection. However, putative relapse infections were detected within and between the three-month timeframe of the single dose administration strategy, warranting reconsideration of the guidelines.

Several studies have employed genetic markers to facilitate identification of $P$. vivax relapse infections $[17,26,30,39,40]$, and WHO has recommended the use of at least one polymorphic marker to aid distinction between homologous and heterologous infections [10]. In this study, we used two types of polymorphic markers, PCR-RFLP size polymorphism in cspr and $m s p 3 a$ and a set of seven microsatellites, to develop criteria for identifying putative relapse infections in southern Mexico. While only two cspr genotypes (vk210 or vk247) were found in our samples, eight alleles were detected for msp3a. Genotyping results from the combination of these two genetic markers appeared to be consistent with those obtained using microsatellites, e.g. sample pairs with the same csprmsp3a genotype also had highly similar microsatellite alleles. Recurrent infections in this low transmission, preelimination setting $[41,42]$ were considered being relapses if: 1$)$ the PI and RI had exactly the same cspr-msp3a genotypes and $>80 \%$ homologous microsatellite alleles; 2 ) the PI and RI had the same rare cspr-msp $3 a$ genotype, and 3) the heterologous RI sample occurred during a nontransmission period when the chance of a new infection was vanishingly small. These criteria allow us to define relapses with a certain degree of confidence, although there is still a possibility of the appearance of new infections with the same genotype during local outbreaks (rapid expansion of parasites with the same genotype), as well as the possibility that mutations can occur during parasite replication within a host, particularly for MS markers that have relatively high mutation rates due to replication slippage. In a recent study, an infection with identical csprmsp1-msp3 and MS genotypes (1-2 MS loci that differs by a single repeat unit) or identical cspr-msp1-msp3 were considered as homologous infection [43]. Our first criterion was similar to those employed by Kim et al. [43]. Because our samples were from a low transmission region, we also considered annual transmission rate and the relatively low possibility of infecting parasites with multiple genotypes in determining relapse or re-infection. However, even though we used nine molecular markers to classify infections in this study, our classifications might miss some heterologous relapses using these criteria. Finally, the methods employed here, compared with, for example, deep-sequencing of all infections, may not identify all genotypes in either the PI or RI.

In southern Chiapas, Mexico, transmission mainly occurs on temperate zone hillsides and is seasonal, once per year generally lasting for 4-6 months; it begins after the rain ends and continues throughout the dry season until the onset of the next rainy season (January-June). Southern Mexico is also a region with low transmission intensity and thus provides an ideal setting for studying relapse in the field because of the low mixed genotype infection rate $(<10 \%)$ and the low likelihood that a patient will receive more than one infective bite within one year $[19,35]$. Our study of 76 recurrent symptomatic infections caused by $P$. vivax in this region showed a large percentage of relapse episodes occurring within the first 52 weeks, based on our definition of relapse, with a majority of these occurring between 27-52 weeks. Most parasites causing recurrent infections $>52$ week after the primary episode were genetically different from the parasites in the PI, suggesting heterologous relapse or re-infections. A few recurrent infections with late intervals $(60,67,81$, and 226 weeks) were also considered to be relapses based on either genetic homology between the PI and RI, and/or occurrence of the RI during the time of year when no known transmission was occurring. Similarly, in El Salvador, 70\% of observed recurrent infections happened within 12 months of the PI, and in the absence of mosquito transmission, and were attributed to relapse [35]. However, it is possible that some heterologous recurrences detected in the high transmission season may be relapses, and some homologous recurrences may correspond to re-infections because the resolution of using a limited number of genetic markers may not be sufficient to identify mixed genotypes or because recurrence infections were derived from parasites of same genotypes (outbreaks).

We found no association between $P$. vivax genotypes and relapse latency, but we cannot entirely rule out this possibility because our sample size was relatively small. Relapse, as defined in the literature, conforms to two 
main patterns: short-latency with relapses occurring mainly within the first 3-6 months after primary infection, as represented by the tropical strain "Chesson" isolated from the Pacific Coast of Asia and those P. vivax parasites found in Thai and Vietnamese [44]. In regions with stable malaria transmission and mild climate, parasites typically exhibit short-latency relapse, as seen in Thailand [45], Colombia [46], French Guyana [47] and Brazil $[48,49]$. In contrast, relapse with a relatively long latency period (7 to 14 months) is represented by the template strain "St Elizabeth" isolated from the Atlantic coast $[5,50,51]$. These criteria are similar to those proposed by Adak based on several $P$. vivax relapse studies in India [32]. The relatively long-term relapses detected here are consistent with relapses observed in other temperate zones [5,6,52]. During 2004-2005, malaria transmission in southern Mexico declined precipitately, with only a few malaria cases reported. This reduction did not lead to a concomitant reduction in parasite genetic diversity based on our microsatellite data (Figure 3) but may have selected for parasites capable of relapsing for longer intervals. In contrast, Hurricane STAN in October, 2005, created an environment that greatly increased parasite transmission, and may have altered relapse patterns in subsequent years. Increases in rainfall can have a dramatic impact on parasite transmission and, in theory, relapse patterns. For example, the incidence of mixed-genotype infections would be expected to increase due to increased parasite transmission. In addition, under conditions of increased transmission, hypnozoite-infected patients are more likely to receive additional bites from infected mosquitoes [36]. In Central America, relapses were reported to occur between 3 and 10 months, with the relapse interval varying between parasite strains and subjects, perhaps indicating adaptation to the unique local ecological environment, including transmission intensity and length of the dry season $[8,19]$. Late interval relapses appear to be less common, but have been documented in other regions, with intervals ranging from between one and two years $[32,53]$, up to four years [54] and even twenty years [55]. In our study, we documented one putative relapse with a 226-week interval ( $>4$ years) containing a single haplotype in both PI and RI samples, and exhibiting perfect genetic homology across all genetic markers. Heterologous relapses were rarely observed in this study, and this may reflect a low rate of mixed genotype infections, typical of a low transmission area. With the exception of patient 2, all relapse episodes were produced by a single $P$. vivax genotype supporting the clonal activation of hypnozoites [18].

\section{Conclusions}

In summary, our study represents the first investigation of $P$. vivax relapse in southern Mexico using molecular markers, and contributes to the global understanding of relapsing malaria. We demonstrate that a large number of putative relapse infections occurred within 52 weeks (one year) of the initial infection, and were largely homologous to the primary infection. They were located mainly in the foothills, which is consistent with observations from other regions with similar temperate zone climate conditions. Genetic markers when analyzed in combination with transmission information can provide a useful tool to characterize recurrent infections.

\section{Additional files}

\begin{abstract}
Additional file 1: Data of $P$. vivax primary and recurrent infections and genetic results.

Additional file 2: Geographic distribution of $P$. vivax recurrent episodes in Southern Chiapas, Mexico. Each circle correspond to one village. Patients with single and genetic homologous primary and recurrent infections are indicated in dark blue. Those with mixed primary infection with a genetic related or unrelated single recurrent infection are in blue and bright blue, respectively. Heterologous recurrent episodes are indicated in white. ${ }^{*}$ indicate one mixed recurrent infection, likely a relapse.

Additional file 3: Temporal distribution of $P$. vivax homologous and heterologous recurrent single infections. Brown arrows indicate homologous PI and RI genotypes and blue arrows indicate heterologous $\mathrm{PI}$ and RI genotypes. Round spot indicate primary blood infection and the arrow head indicate the recurrent blood infection. Thick arrows indicate rare msp3a genotypes. Patient number is indicated at the arrow side. Red line indicate cut-off value for low transmission setting.
\end{abstract}

\section{Abbreviations}

CQ: Chloroquine; PQ: Primaquine; PI: Primary infection; RI: Recurrent infection; csp: Circumsporozoite protein; msp3a: Merozoite surface protein 3 alpha; MS: Microsatellites; PCR: Polymerase chain reaction; RFLP: Restriction fragment length polymorphism.

\section{Competing interests}

The authors declare no competing interests.

\section{Authors' contributions}

LGC participated in the conception and design of the study, analysis and interpretation of data, preparing tables and figures, and manuscript writing; JM participated in microsatellite analysis and manuscript writing; GC and FSV carried out the PCR-RFLP analysis of genes csp and msp3a; MS participated in patient follow-up in the field and collection of $P$. vivax samples, and preparation of figures; DJ participated in microsatellite analyses; preparing figures and tables, interpretation of data and manuscript writing; XS participated in interpretation of data and manuscript writing; RT and VCH prepared a data base of the 10-year malaria case report and data analysis. All authors read and approved the final manuscript.

\section{Acknowledgments}

This work was supported by funds of CONACYT salud-2004-119 and CB2009-01-131247, and by the Divisions of Intramural Research at the National Institute of Allergy and Infectious Diseases. We thank the technical assistance of J.A. Nettel in DNA extraction and patient handling.

\section{Author details}

${ }^{1}$ CRISP, National Institute for Public Health, Tapachula, Chiapas 30700, Mexico. ${ }^{2}$ Laboratory of Malaria and Vector Research, NIAID, National Institutes of Health, Bethesda, MD 20892, USA. ${ }^{3}$ Sanitary Jurisdiction VII, Ministry of Health, Tapachula, Chiapas, Mexico.

Received: 3 September 2012 Accepted: 3 April 2013

Published: 18 April 2013 


\section{References}

1. Centro Nacional de Vigilancia Epidemiológica y Control de Enfermedades: Dirección general de epidemiología: Secretaria de Salud México. http://www. dgepi.salud.gob.mx/2010/plantilla/intd_boletin.html.

2. Betanzos AF: La malaria en México. Progresos y desafíos hacia su eliminación Challenges and progress in the elimination of malaria in Mexico [Challenges and progress in the elimination of malaria in Mexico. Bol Med Hosp Infant Mex 2011, 68(2):159-168.

3. Cox FE: History of the discovery of the malaria parasites and their vectors. Parasit Vectors 2010, 3(1):5.

4. Baird JK, Hoffman SL: Primaquine therapy for malaria. Clin Infect Dis 2004, 39(9):1336-1345.

5. White NJ: Determinants of relapse periodicity in Plasmodium vivax malaria. Malar J 2011, 10:297.

6. Verhave JP: Experimental, therapeutic and natural transmission of Plasmodium vivax tertian malaria: scientific and anecdotal data on the history of Dutch malaria studies. Parasit Vectors 2013, 6(1):19.

7. Contacos PG, Collins WE: Letter: Malarial relapse mechanism. Trans $R$ Soc Trop Med Hyg 1973, 67(4):617-618.

8. Contacos PG, Collins WE, Jeffery GM, Krotoski WA, Howard WA: Studies on the characterization of Plasmodium vivax strains from Central America. Am J Trop Med Hyg 1972, 21(5):707-712.

9. Hill DR, Baird JK, Parise ME, Lewis LS, Ryan ET, Magill AJ: Primaquine: report from CDC expert meeting on malaria chemoprophylaxis I. Am J Trop Med Hyg 2006, 75:402-415.

10. WHO (Ed): Guidelines for malaria treatment. Secondth edition. Geneva; 2010. http://www.who.int/malaria/publications/atoz/9789241547925/en/index.html.

11. Baird JK, Schwartz E, Hoffman SL: Prevention and treatment of vivax malaria. Curr Infect Dis Rep 2007, 9(1):39-46.

12. Goller JL, Jolley D, Ringwald P, Biggs BA: Regional differences in the response of Plasmodium vivax malaria to primaquine as anti-relapse therapy. Am J Trop Med Hyg 2007, 76(2):203-207.

13. Spudick JM, Garcia LS, Graham DM, Haake DA: Diagnostic and therapeutic pitfalls associated with primaquine-tolerant Plasmodium vivax. I Clin Microbiol 2005, 43(2):978-981.

14. Secretaria de Salud: NORMA Oficial Mexicana NOM-032-SSA2-2002, Para lo vigilancia epidemiológica, prevención y control de enfermedades transmitidas por vector. México; 2002. http://www.salud.gob.mx/unidades/cdi/nom/ 032ssa202.html.

15. Mendez Galvan JF, Guerrero Alvarado J, Gonalez Mora M, Perez Landa M, Quintero Cabanillas R: Evaluation of an alternative scheme of treatment for the control of malaria. Salud Publica Mex 1984, 26(6):561-572.

16. WHO: World Malaria Report. Geneva; 2009. http://www.who.int/malaria/ world_malaria_report_2009/en/index.html.

17. Craig AA, Kain KC: Molecular analysis of strains of Plasmodium vivax from paired primary and relapse infections. J Infect Dis 1996, 174(2):373-379.

18. Chen N, Auliff A, Rieckmann K, Gatton M, Cheng Q: Relapses of Plasmodium vivax infection result from clonal hypnozoites activated at predetermined intervals. J Infect Dis 2007, 195(7):934-941.

19. Imwong M, Snounou G, Pukrittayakamee S, Tanomsing N, Kim JR, Nandy A, Guthmann JP, Nosten F, Carlton J, Looareesuwan S, et al: Relapses of Plasmodium vivax infection usually result from activation of heterologous hypnozoites. J Infect Dis 2007, 195(7):927-933.

20. Gonzalez-Ceron L, Rodriguez MH, Nettel JC, Villarreal C, Kain KC, Hernandez JE: Differential susceptibilities of Anopheles albimanus and Anopheles pseudopunctipennis to infections with coindigenous Plasmodium vivax variants VK210 and VK247 in southern Mexico. Infect Immun 1999, 67(1): 410-412.

21. Gonzalez-Ceron L, Rodriguez MH, Santillan F, Chavez B, Nettel JA, Hernandez-Avila JE, Kain KC: Plasmodium vivax: ookinete destruction and oocyst development arrest are responsible for Anopheles albimanus resistance to circumsporozoite phenotype VK247 parasites. Exp Parasitol 2001, 98(3):152-161.

22. Gonzalez-Ceron L, Rodriguez MH, Chavez-Munguia B, Santillan F, Nettel JA, Hernandez-Avila JE: Plasmodium vivax: impaired escape of Vk210 phenotype ookinetes from the midgut blood bolus of Anopheles pseudopunctipennis. Exp Parasitol 2007, 115(1):59-67.

23. Zakeri S, Barjesteh H, Djadid ND: Merozoite surface protein-3alpha is a reliable marker for population genetic analysis of Plasmodium vivax. Malar J 2006, 5:53.
24. Galinski MR, Corredor-Medina C, Povoa M, Crosby J, Ingravallo P, Barnwell JW: Plasmodium vivax merozoite surface protein-3 contains coiled-coil motifs in an alanine-rich central domain. Mol Biochem Parasitol 1999 101(1-2):131-147.

25. Rayner JC, Corredor V, Feldman D, Ingravallo P, Iderabdullah F, Galinski MR, Barnwell JW: Extensive polymorphism in the Plasmodium vivax merozoite surface coat protein MSP-3alpha is limited to specific domains. Parasitology 2002, 125(Pt 5):393-405.

26. Cui L, Mascorro CN, Fan Q, Rzomp KA, Khuntirat B, Zhou G, Chen H, Yan G, Sattabongkot J: Genetic diversity and multiple infections of Plasmodium vivax malaria in Western Thailand. Am J Trop Med Hyg 2003, 68(5):613-619.

27. Joy DA, Gonzalez-Ceron L, Carlton JM, Gueye A, Fay M, McCutchan TF, Su XZ: Local adaptation and vector-mediated population structure in Plasmodium vivax malaria. Mol Biol Evol 2008, 25(6):1245-1252.

28. Kain KC, Brown AE, Webster HK, Wirtz RA, Keystone JS, Rodriguez MH, Kinahan J, Rowland M, Lanar DE: Circumsporozoite genotyping of global isolates of Plasmodium vivax from dried blood specimens. J Clin Microbiol 1992, 30(7):1863-1866

29. Imwong M, Pukrittayakamee S, Gruner AC, Renia L, Letourneur F, Looareesuwan S, White NJ, Snounou G: Practical PCR genotyping protocols for Plasmodium vivax using Pvcs and Pvmsp1. Malar J 2005, 4(1):20.

30. Bruce MC, Galinski MR, Barnwell JW, Snounou G, Day KP: Polymorphism at the merozoite surface protein-3alpha locus of Plasmodium vivax: global and local diversity. Am J Trop Med Hyg 1999, 61(4):518-525.

31. Kim JR, Imwong M, Nandy A, Chotivanich K, Nontprasert A, Tonomsing N, Maji A, Addy M, Day NP, White NJ, et al: Genetic diversity of Plasmodium vivax in Kolkata. India. Malar J 2006, 5:71.

32. Adak T, Valecha N, Sharma VP: Plasmodium vivax polymorphism in a clinical drug trial. Clin Diagn Lab Immunol 2001, 8(5):891-894.

33. Peakall R, Smouse PE: GenAlEx 6.5: genetic analysis in Excel. Population genetic software for teaching and research--an update. Bioinformatics 2012, 28(19):2537-2539.

34. Koepfli C, Mueller I, Marfurt J, Goroti M, Sie A, Oa O, Genton B, Beck HP, Felger I: Evaluation of Plasmodium vivax genotyping markers for molecular monitoring in clinical trials. J Infect Dis 2009, 199(7):1074-1080

35. Mason J: Patterns of Plasmodium vivax recurrence in a high-incidence coastal area of El Salvador, C. A. Am J Trop Med Hyg 1975, 24(4):581-585.

36. Hulden L, Heliovaara K: Natural relapses in vivax malaria induced by Anopheles mosquitoes. Malar J 2008, 7:64

37. Rodriguez MH, Betanzos-Reyes AF, Hernandez-Avila JE, Mendez-Galvan JF, Danis-Lozano R, Altamirano-Jimenez A: The participation of secondary clinical episodes in the epidemiology of vivax malaria during pre- and post-implementation of focal control in the state of Oaxaca. Mexico. Am $J$ Trop Med Hyg 2009, 80(6):889-895.

38. Lin E, Kiniboro B, Gray L, Dobbie S, Robinson L, Laumaea A, Schopflin S, Stanisic D, Betuela I, Blood-Zikursh M, et al: Differential patterns of infection and disease with $P$. falciparum and $P$. vivax in young Papua New Guinean children. PLoS One 2010, 5(2):e9047.

39. Hanna JN, Ritchie SA, Eisen DP, Cooper RD, Brookes DL, Montgomery BL: An outbreak of Plasmodium vivax malaria in Far North Queensland, 2002. Med J Aust 2004, 180(1):24-28.

40. Havryliuk T, Ferreira MU: A closer look at multiple-clone Plasmodium vivax infections: detection methods, prevalence and consequences. Mem Inst Oswaldo Cruz 2009, 104(1):67-73.

41. Rodriguez MH, Betanzos-Reyes AF: Plan to improve malaria control towards its elimination in Mesoamerica. Salud Publica Mex 2011, 53(3):S333-348.

42. WHO: Malaria elimination. A field manual for low and moderate endemic countries. Geneva; 2007. http://whalibdoc.who.int/publications/2007/ 9789241596084 eng.pdf.

43. Kim JR, Nandy A, Maji AK, Addy M, Dondorp AM, Day NP, Pukrittayakamee S, White NJ, Imwong M: Genotyping of Plasmodium vivax reveals both short and long latency relapse patterns in Kolkata. PLOS One 2012, 7(7):e39645.

44. Cogswell FB: The hypnozoite and relapse in primate malaria. Clin Microbiol Rev 1992, 5(1):26-35.

45. Bunnag D, Karbwang J, Thanavibul A, Chittamas S, Ratanapongse Y, Chalermrut K, Bangchang KN, Harinasuta T: High dose of primaquine in primaquine resistant vivax malaria. Trans R Soc Trop Med Hyg 1994, 88(2):218-219. 
46. Carmona-Fonseca J, Maestre A: Prevention of Plasmodium vivax malaria recurrence: efficacy of the standard total dose of primaquine administered over 3 days. Acta Trop 2009, 112(2):188-192.

47. Hanf M, Stephani A, Basurko C, Nacher M, Carme B: Determination of the Plasmodium vivax relapse pattern in Camopi. French Guiana Malar J 2009, 8:278.

48. Boulos M, Amato Neto V, Dutra AP, Di Santi SM, Shiroma M: Frequency of malaria relapse due to Plasmodium vivax in a non-endemic region (Sao Paulo, Brazil). Rev Inst Med Trop Sao Paulo 1991, 33(2):143-146.

49. Orjuela-Sanchez $P$, da Silva NS, da Silva-Nunes M, Ferreira MU: Recurrent parasitemias and population dynamics of Plasmodium vivax polymorphisms in rural Amazonia. Am J Trop Med Hyg 2009, 81(6):961-968.

50. Coatney GR, Cooper WC, Young MD: Studies in human malaria. XXX. A summary of 204 sporozoite-induced infections with the Chesson strain of Plasmodium vivax. J Natl Malar Soc 1950, 9(4):381-396.

51. White NJ: Malaria. In Manson's Tropical Diseases. 21st edition. Edited by Cook GC, Zumla Al. London: WB Sounders; 2003:1205-1295.

52. Moon SU, Lee HW, Kim JY, Na BK, Cho SH, Lin K, Sohn WM, Kim TS: High frequency of genetic diversity of Plasmodium vivax field isolates in Myanmar. Acta Trop 2009, 109(1):30-36.

53. Haghdoost AA, Mazhari S, Bahaadini K: Estimating the relapse risk of Plasmodium vivax in Iran under national chemotherapy scheme using a novel method. J Vector Borne Dis 2006, 43(4):168-172.

54. Mangoni AA, Jackson SH: Age-related changes in pharmacokinetics and pharmacodynamics: basic principles and practical applications. $\mathrm{Br} J \mathrm{Clin}$ Pharmacol 2004, 57(1):6-14.

55. Maslin J, Cuguillere A, Bonnet D, Martet G: Malaria attack: a very late relapse due to Plasmodium vivax. Bull Soc Pathol Exot 1997, 90(1):25-26.

doi:10.1186/1756-3305-6-109

Cite this article as: Gonzalez-Ceron et al:: Molecular and epidemiological characterization of Plasmodium vivax recurrent infections in southern Mexico. Parasites \& Vectors 2013 6:109.

\section{Submit your next manuscript to BioMed Central and take full advantage of:}

- Convenient online submission

- Thorough peer review

- No space constraints or color figure charges

- Immediate publication on acceptance

- Inclusion in PubMed, CAS, Scopus and Google Scholar

- Research which is freely available for redistribution 